\title{
The role of T-box genes in the tumorigenesis and progression of cancer (Review)
}

\author{
FANGYUAN CHANG ${ }^{1,2}$, PEIPEI XING ${ }^{1,2}$, FENGJU SONG $^{2,3}$, XIAOLING DU ${ }^{4}$, \\ GUOWEN WANG ${ }^{1,2}$, KEXIN CHEN $^{2,3}$ and JILONG YANG ${ }^{1,2}$
}

\author{
${ }^{1}$ Bone and Soft Tissue Tumor Department; ${ }^{2}$ National Clinical Research Center for Cancer; \\ ${ }^{3}$ Epidemiology and Biostatistics Department, Tianjin Medical University Cancer Institute \& Hospital, \\ Tianjin 300060; ${ }^{4}$ Department of Diagnostics, Tianjin Medical University, Tianjin 300061, P.R. China
}

Received July 18, 2015; Accepted September 9, 2016

DOI: $10.3892 / 01.2016 .5296$

\begin{abstract}
The T-box (TBX) genes are part of an evolutionarily conserved family of transcription factors involved in organ development. They serve key roles in a number of molecular mechanisms, including proliferation, cell fate and organ identity. In addition, previous studies suggest that TBX genes have essential functions in the tumorigenesis and progression of various types of cancer. For example, TBX proteins served significant roles in carcinogenesis, proliferation and differentiation, senescence and apoptosis, invasion and migration, mesenchymal-epithelial and epithelial-mesenchymal transition, oncogenic signaling pathways and drug sensitivity. However, the exact mechanisms by which TBX genes carry out these functions have not yet been fully elucidated. The present review focuses on the role of TBX genes in cancer, with the aim of further clarifying their function. As altered levels of TBX proteins have detrimental consequences in numerous types of cancer, there is a need for further research into TBX genes, which this review may aid through providing a comprehensive insight into the topic.
\end{abstract}

Correspondence to: Dr Jilong Yang, Bone and Soft Tissue Tumor Department, Tianjin Medical University Cancer Institute \& Hospital, Huanhuxi Road, Hexi, Tianjin 300060, P.R. China

E-mail: yangjilong@tjmuch.com

Abbreviations: B-RAF, B-Raf proto-oncogene; PMA, phorbol-12-myristate-13-acetate; AP-1, activator protein 1; PML, promyelocytic leukemia; CDK, cyclin-dependent kinase; MDM2, MDM2 proto-oncogene; EGR, early growth response protein; NDRG1, N-myc downregulated gene 1; HDAC, histone deacetylase; CDKN2A, cyclin-dependent kinase inhibitor 2A; PSCA, prostate stem cell antigen; PIWIL1, piwi-like RNA-mediated gene silencing 1; PTEN, phosphatase and tensin homolog; ATM, ATM serine/threonine kinase; CHK2, checkpoint kinase 2; AKT3: Akt serine/threonine kinase 3; siRNA, small interfering RNA

Key words: T-box genes, cancer, tumorigenesis, tumor progression, therapy

\section{Contents}

1. Introduction

2. TBX genes are involved in tumor cell invasion and migration

3. TBX genes are involved in tumor cell immortality and proliferation

4. TBX genes are involved in tumorigenesis

5. TBX genes are involved in EMT

6. TBX genes are involved in tumorigenic signaling pathways

7. TBX genes as targets for cancer therapy

8. Conclusion

\section{Introduction}

The T-box (TBX) gene family is an evolutionarily ancient gene family, as indicated by phylogenetic analysis (1), and has been widely studied in the field of developmental biology (2). TBX genes serve key roles during organogenesis and pattern formation in vertebrate and invertebrate embryos (3). Previous studies have shown that TBX genes encode a group of transcription factors that are characterized by a highly conserved DNA-binding domain and an unusual mode of DNA recognition (4-7). Certain TBX genes are purely activators or repressors, while others contain activation and repression domains (8). Since the first TBX family member was discovered in 1927, the TBX family has been increasingly implicated in the development of various organs and the pathogenesis of a number of syndromes $(9,10)$.

Over 20 TBX genes have been identified in various species, ranging from invertebrates, including Drosophila and Caenorhabditis elegans, to vertebrates, including zebrafish, Xenopus, mice, chickens and humans (11-13). Current phylogenetic analysis divides the TBX gene family into five subfamilies, T, Tbx1, Tbx2, Tbx6 and Tbr1 $(8,14)$. The $\mathrm{T}$ subfamily includes $\mathrm{T}$ and TBX19; the Tbx 1 subfamily includes TBX1, TBX10, TBX15, TBX18, TBX20 and TBX22; the Tbx2 subfamily includes TBX2, TBX3, TBX4 and TBX5; the Tbx6 subfamily includes TBX6 and Mga; and the Tbr1 subfamily includes TBR1, TBR2 and TBX21. TBX genes serve significant roles in craniofacial (TBR1, TBX10, TBX15 and TBX22), brain (TBR1 and TBR2), mammary gland (TBX2 and TBX3), pituitary gland (TBX3, TBX19), thymus (TBX1), 
liver (TBX3), lung (TBX2, TBX4 and TBX5) and limb (TBX4 and TBX5) development, in addition to pigmentation (TBX15) and the immune system (TBX21) $(8,14)$. Furthermore, TBX proteins function in proliferation, differentiation, tissue integrity and epithelial-mesenchymal transition (EMT) $(8,14)$.

Recent studies have shown that defects in, or overexpression of, certain TBX genes may be involved in the genesis and progression of a variety of types of cancer $(2,15)$. For example, unlike the majority of members of the TBX family, which function as transcriptional activators, TBX2 and TBX3 are the only mammalian TBX factors that function as transcriptional repressors $(16,17)$. The role of TBX2 and TBX3 in the oncogenic process is thought to be associated with an increase in their level of expression, as they have been found to be overexpressed in 8 different types of cancer, including breast, cervical, ovarian, pancreatic, liver and bladder cancer $(18,19)$. However, there is no comprehensive summary of the molecular and mechanistic changes to TBX genes during the genesis and progression of cancer. Therefore, in the present review, the roles of TBX genes in cancer are discussed. Through increased understanding of TBX factors at a genetic and molecular level, targeted therapy of these factors may become a promising therapy for the treatment of cancer.

\section{TBX genes are involved in tumor cell invasion and mi- gration}

Preliminary evidence suggests that overexpression of the TBX3 gene is associated with several types of cancer, and that increased levels of TBX3 are associated with the promotion of tumor migration and invasion (20). Peres et al (21) reported that TBX3 knockdown in MCF-7 breast cancer cells and in metastatic melanoma cells resulted in increased proliferation and reduced migration. Boyd et al (22) applied a genome-wide expression profiling approach in an attempt to identify an association between the expression of B-Raf proto-oncogene (B-RAF), the transcriptional repressor TBX3 and E-cadherin. The results of this study demonstrated that B-Raf induced the expression of TBX3, which potently repressed E-cadherin expression (Fig. 1). Therefore, TBX3 may act as a critical regulator of the oncogenic B-Raf signaling pathway and as a promoter of metastasis in B-RAF-mutant melanomas (22). Furthermore, a previous study has shown that overexpression of TBX2 and TBX3 in melanoma cells downregulated endogenous E-cadherin expression, whereas depletion of TBX3, but not TBX2, increased E-cadherin mRNA and protein levels, and decreased melanoma invasion in vitro (23). Consistent with these observations, TBX3 and E-cadherin expression are inversely correlated in melanoma tissue.

A previous study has shown that the phorbolester phorbol-12-myristate-13-acetate (PMA) increases TBX3 protein and mRNA levels in a protein kinase $\mathrm{C}$-dependent manner, via activator protein 1 (AP-1) (24). Furthermore, AP-1 mediated activation of the TBX3 gene by binding to a non-consensus PMA-response element in the TBX3 promoter in vitro and in vivo (24). This demonstrates that TBX3 contributes to PMA-induced cell migration, which has previously been observed in the MCF-7 breast epithelium cancer cell line (25). These results provide evidence that increased levels of TBX3 contribute to tumor cell migration (Fig. 1).

\section{TBX genes are involved in tumor cell immortality and proliferation}

TBX genes and promyelocytic leukemia (PML). The tumor-suppressing function of PML was identified through the observation that PML knockout mice were more tumor-prone (26). PML protein levels were found to be significantly reduced in a cancers of different histologic origins, including prostate and colon adenocarcinomas, breast and lung carcinomas, lymphomas, and central nervous system and germ cell tumors, compared with corresponding normal tissue (27). Conversely, excessive TBX2 protein levels have been detected in primary human breast and pancreatic cancer, and in various cancer cell lines (2). This indicates that there is an inverse correlation between TBX2 and PML protein levels in cancer. Through the combination of gene expression profiling, chromatin-binding analysis and promoter-reporter studies, Martin et al (28) identified TBX2 as a novel and direct PML-repressible E2F-target gene involved in the evasion of senescence. TBX2 gene repression might actively contribute to senescence, as depletion of TBX2 triggered PML and caused cells to enter senescence (28). Reciprocally, elevated TBX2 levels antagonized the pro-senescence functions of PML through direct protein-protein interaction. These observations suggest that PML and TBX2 act in an autoregulatory loop to mediate the effective execution of senescence (Fig. 1).

TBX genes, $p 14^{A R F}$ and $p 21^{C I P I}$. During mouse oncogenesis, TBX2 and TBX3 have been associated with the repression of tumor suppressor genes $\mathrm{p} 19^{\mathrm{ARF}}$ (p14 ${ }^{\mathrm{ARF}}$ in humans) and the cyclin-dependent kinase (CDK) inhibitor p21 ${ }^{\mathrm{CIP1}}(18)$. p14 ${ }^{\mathrm{ARF}}$ increases the level of $\mathrm{p} 53$ by elevating the level of MDM2 proto-oncogene (MDM2). Increased p53 levels lead to increased levels of $\mathrm{p} 21^{\mathrm{CIP} 1}$, and subsequent cell cycle arrest in $\mathrm{G} 1$ and G2/M (29,30). A further study demonstrated that p14 ${ }^{\mathrm{ARF}}$ expression could be induced by overexpression of various oncogenes or by mitogenic signals (31). p14 ${ }^{\mathrm{ARF}}$ can bind to MDM2, causing stabilization of p53, which initiates a program of gene expression leading to cell cycle arrest or apoptosis (31). Activated p53, which can initiate growth arrest in senescent cells, causes activation of $\mathrm{p} 21^{\mathrm{CIP} 1}$, which is necessary for p53-mediated growth arrest (31). TBX2 and TBX3 suppressed senescence efficiently in a mouse model through the inhibition of $\mathrm{p} 19^{\mathrm{ARF}}$ expression (31). Then, inhibition of p19 ${ }^{\mathrm{ARF}}$ expression reduced the level of MDM2, blocking stabilization of p53 and thus inhibiting p53-mediated upregulation of $\mathrm{p} 21^{\mathrm{CIP1}}$, which has been implicated in the control of proliferation, differentiation and senescence (31). Therefore, it may be necessary for the $\mathrm{p} 14^{\mathrm{ARF}} / \mathrm{p} 19^{\mathrm{ARF}}-\mathrm{MDM} 2-\mathrm{p} 53-\mathrm{p} 21$ signaling pathway (Fig. 1), a well-characterized mechanism of cellular senescence, to be targeted at multiple points for the treatment of cancer.

TBX genes and early growth response (EGR) 1 . The EGR protein family includes zinc-finger transcription factors involved in cell proliferation and apoptosis. EGR1 is best characterized as a direct regulator of a number of signaling pathways, it has significant tumor-suppressing properties, including the promotion of apoptosis in response to stress and DNA damage in a number of types of cancer $(32,33)$. Redmond et al (34) described a novel 


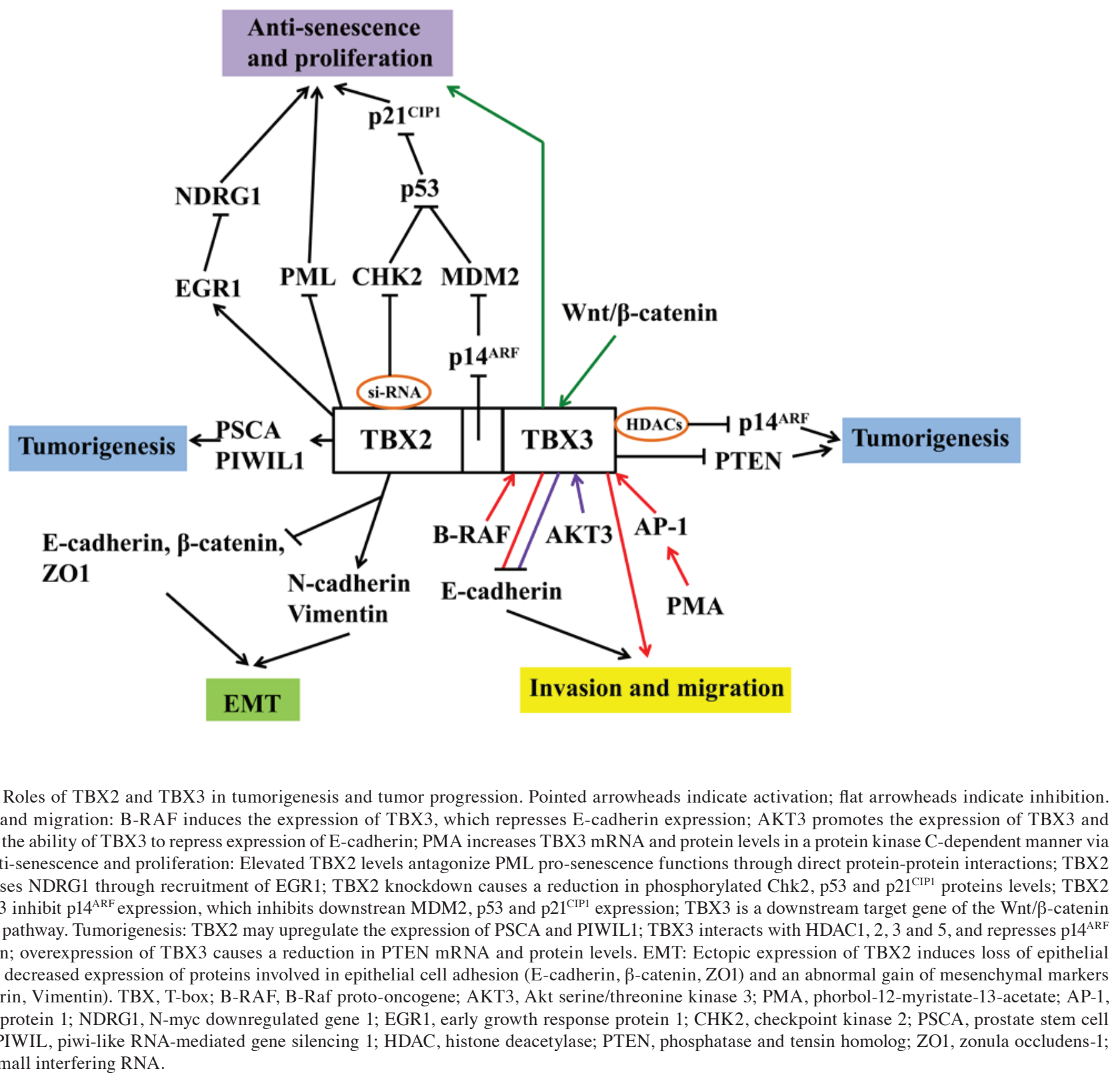

Figure 1. Roles of TBX2 and TBX3 in tumorigenesis and tumor progression. Pointed arrowheads indicate activation; flat arrowheads indicate inhibition. Invasion and migration: B-RAF induces the expression of TBX3, which represses E-cadherin expression; AKT3 promotes the expression of TBX3 and enhances the ability of TBX3 to repress expression of E-cadherin; PMA increases TBX3 mRNA and protein levels in a protein kinase C-dependent manner via AP-1. Anti-senescence and proliferation: Elevated TBX2 levels antagonize PML pro-senescence functions through direct protein-protein interactions; TBX2 co-represses NDRG1 through recruitment of EGR1; TBX2 knockdown causes a reduction in phosphorylated Chk2, p53 and p21 ${ }^{\text {CIP1 }}$ proteins levels; TBX2 and TBX3 inhibit p14 ${ }^{\mathrm{ARF}}$ expression, which inhibits downstrean MDM2, p53 and p21 ${ }^{\mathrm{CIP1}}$ expression; TBX3 is a downstream target gene of the Wnt/ $\beta$-catenin signaling pathway. Tumorigenesis: TBX2 may upregulate the expression of PSCA and PIWIL1; TBX3 interacts with HDAC1, 2, 3 and 5, and represses p14 ${ }^{\mathrm{ARF}}$ expression; overexpression of TBX3 causes a reduction in PTEN mRNA and protein levels. EMT: Ectopic expression of TBX2 induces loss of epithelial adhesion, decreased expression of proteins involved in epithelial cell adhesion (E-cadherin, $\beta$-catenin, ZO1) and an abnormal gain of mesenchymal markers (N-cadherin, Vimentin). TBX, T-box; B-RAF, B-Raf proto-oncogene; AKT3, Akt serine/threonine kinase 3; PMA, phorbol-12-myristate-13-acetate; AP-1, activator protein 1; NDRG1, N-myc downregulated gene 1; EGR1, early growth response protein 1; CHK2, checkpoint kinase 2; PSCA, prostate stem cell antigen; PIWIL, piwi-like RNA-mediated gene silencing 1; HDAC, histone deacetylase; PTEN, phosphatase and tensin homolog; ZO1, zonula occludens-1; siRNA, small interfering RNA.

mechanism of TBX2 interaction with EGR1, which drove cell proliferation and survival in breast cancer cell lines. Depletion of TBX2 or EGR1 resulted in growth inhibition, and the expression of cell senescence and apoptotic markers, including deleted in esophageal cancer 1 (Dec1), p21 $1^{\mathrm{CIP1}}$ and p53 (34). The putative breast cancer suppressor, $\mathrm{N}$-myc downregulated gene 1 (NDRG1), is implicated in cell differentiation, apoptosis and senescence (34). TBX2 was found to repress NDRG1 not through direct promoter interaction, but as a corepressor through recruitment of EGR1. In this way, TBX2 drove the proliferation of breast cancer cells (34). NDRG1 was demonstrated to be a significant effector of growth control downstream of TBX2-EGR1, and its expression recapitulated growth inhibition and induced expression of the cell senescence marker Dec1 (34). These results demonstrate the significance of the EGR1 signaling pathway in cancer (Fig. 1) and reveal it to be a potential novel therapeutic target.

TBX genes, histone deacetylase (HDAC) 1 and p21. Rhabdomyosarcomas (RMSs) are the most prevalent soft tissue sarcomas in children and share many features with developing skeletal muscle tissue. A recent study by Zhu et al (35) identified that TBX2 was highly upregulated in tumor cells of the major RMS subtypes. The results of this study demonstrated that elevated expression of TBX2 contributes to the pathology of RMS cells. TBX2 inhibited the regulatory factors myogenic differentiation 1 and myogenin, leading to the promotion of proliferation (35). Furthermore, TBX2 recruited the histone deacetylase HDAC1 and inhibited p21, which led to increased proliferation and decreased differentiation-specific gene expression (35). Depletion or inhibition of TBX2 completely inhibited tumor cell growth in RMS cells (35). These results indicate that deregulated TBX2 serves as an oncogene in RMS, and may be a novel diagnostic marker and/or therapeutic target in patients with RMS.

The CDK inhibitor 2A (CDKN2A) locus, which encodes p1 $6^{\mathrm{INK} 4 \mathrm{~A}}$ and $\mathrm{p} 14^{\mathrm{ARF}}$, promotes senescence (36). A previous study found that CDKN2A-null B16 cells exhibited a specific deletion at the CDKN2A locus, leading to loss of function of $\mathrm{p} 16^{\mathrm{INK} 4 \mathrm{~A}}$ and 
p14 ${ }^{\mathrm{ARF}}$ (37). In addition, the activation of a dominant-negative TBX2 (dnTBX2) gene induced senescence in the CDKN2A-null B16 melanoma cells (37). Furthermore, senescence was accompanied by increased expression of the TBX2 target gene p21 and displacement of HDAC1 from the p21 promoter, which markedly reduced proliferation (37). This TBX2-mediated molecular mechanism of transcriptional repression suggests that continued TBX2 activity is required to prevent CDKN2A-independent senescence in transformed cells.

Holt-Oram syndrome is characterized by upper limb malformations and cardiac septation defects (38). Mutations in the TBX5 gene were found to cause Holt-Oram syndrome in humans (38). In addition, a previous study reported that ectopic expression of TBX5 induced apoptosis and decreased the growth rate of U2OS cells, indicating that TBX5 is a novel regulator of apoptosis and cell growth (39). These results suggest that the TBX5 gene and protein serve important roles in the development of certain tissues.

\section{TBX genes are involved in tumorigenesis}

Liu et al (40) investigated the expression and association of prostate stem cell antigen (PSCA), piwi-like RNA-mediated gene silencing 1 (PIWIL1) and TBX2 in endometrial adenocarcinoma (EAC). Elevated expression of PSCA, PIWIL1 and TBX2 was observed significantly more frequently in EACs compared with normal endometrium (40). TBX2 expression was positively correlated with PSCA and PIWIL1 expression (40), indicating that TBX2 may upregulate PSCA and PIWIL1 (Fig. 1). The association between clinicopathological features and the three genes (40) revealed that they may have roles in the development and progression of EAC, and that PIWIL1 and TBX2 may be candidate markers for early pathological diagnosis and detection. PSCA, PIWIL1 and TBX2 were not associated with cancer stage (40), suggesting an involvement in the initiation of EAC rather than invasion. However, PSCA and TBX2 were associated with lymph node metastasis, and may be candidate targets for EAC prognosis and therapy (40). This study provides a basis for further investigations into the carcinogenesis of EAC.

HDACs serve an essential role in transcriptional regulation (41-44) and have been found to be overexpressed in a number of malignancies, including breast cancer $(45,46)$. The results of a study by Yarosh et al (47) indicated that overexpression of TBX3 increases the degradation of p53 via downregulating $\mathrm{p} 14^{\mathrm{ARF}}$ expression, leading to the promotion of breast cancer tumorigenesis. TBX3 was found to interact with HDAC1, 2, 3 and 5 to repress their downstream effects on gene expression (47). In the MCF-7 breast cancer cell line, HDACs were tested for their ability to reverse TBX3 regulation of p14 ${ }^{\mathrm{ARF}}$ in a dose-dependent manner (47). TBX3-mediated gene repression may function by recruiting HDACs to the T-box binding site in the promoter region of genes and was dependent on HDAC activity (47). The results of this study suggest that TBX3-HDAC interaction is important in breast cancer development (47). Therefore, HDACs may have an anticancer effect through TBX3-HDAC interaction (Fig. 1).

A previous study reported that $\mathrm{mRNA}$ and protein levels of TBX3 were increased in head and neck squamous cell carcinoma (HNSCC) samples compared with their normal tissue counterparts (48), and another study found that phosphatase and tensin homolog (PTEN) mRNA levels were decreased in HNSCC tissues (49). Furthermore, overexpression of TBX3 in human HeLa and HEK cell lines was demonstrated to cause a reduction in endogenous PTEN mRNA and protein levels (48). In addition, transcription activity assays have shown that TBX3 is capable of repressing basal and induced activity of PTEN (48). These results suggest that TBX3 represses PTEN and is overexpressed in HNSCC (Fig. 1).

\section{TBX genes are involved in EMT}

EMT has been increasingly recognized as a key step in the progression of primary tumors into metastases (50). EMT interrupts cell-to-cell contact in a homocellular manner in tumors, allowing the dissemination of cells from the primary site (51). During tumor progression, the EMT process appears to be triggered by genes typically expressed in the early embryo, including TWIST, SNAIL, SLUG, GOOSECOID and SIP1 (52-55), and targeting them may prevent tumor invasion and metastasis.

Shimoda et al (51) reported that metastatic ACCS-M green fluorescent protein cells (ACCS-M GFP cell line), established from AdCC cells, demonstrated characteristics of EMT, exhibited sphere-forming ability, and had high expression levels of EMT-related genes and stem cell and differentiation markers. These observations suggest that ACCS-M ${ }^{\mathrm{GFP}+}$ cells show characteristics of cancer stem-like cells (CSCs), which may be involved in the EMT of adenoid cystic carcinoma (AdCC) cells. Surprisingly, short hairpin RNA silencing of the TBX transcription factor, $\mathrm{T}$, resulted in downregulation of EMT and CSC markers in clinical samples of AdCC (51). Notably, sphere-forming ability, EMT characteristics and tumorigenicity were simultaneously lost (51). Therefore, $\mathrm{T}$ may be a regulator of CSC marker expression and EMT in AdCC cells. In addition, $\mathrm{T}$ may be a potential therapeutic target for future anti-CSC treatment for AdCC.

Wang et al (56) reported that ectopic expression of TBX2 in wild-type $\mathrm{HC} 11$ and MCF10A mammary epithelial cells induced morphological, molecular and behavioral changes characteristic of EMT. This included loss of expression of proteins involved in epithelial cell adhesion (E-cadherin, $\beta$-catenin and zonula occludens 1), abnormal gain of mesenchymal markers (N-cadherin, vimentin), and increased cell motility and invasion (56). Conversely, depletion of overexpressed endogenous TBX2 in the malignant human breast carcinoma cell lines MDA-MB-435 and MDA-MB-157 led to the restoration of epithelial characteristics and loss of mesenchymal markers (56). In addition, chromatin immunoprecipitation (ChIP) analysis and cell-based reporter assays revealed that TBX2 directly repressed transcription of E-cadherin, a tumor suppressor gene, the loss of which is essential for malignant tumor progression (56). The results of this study demonstrated an unanticipated link between TBX2 deregulation in cancer, and the acquisition of EMT and invasive features in epithelial tumor cells (Fig. 1).

\section{TBX genes are involved in tumorigenic signaling path- ways}

ATM serinelthreonine kinase (ATM)-checkpoint kinase 2 (CHK2)-p53 signaling pathway. Wansleben et al (57) 
demonstrated that silencing of TBX2 reversed several features of transformation in breast cancer and melanoma cells. In addition, it was shown that ectopic expression of TBX2 resulted in genetically unstable polyploidy cells with resistance to cisplatin (57). In an attempt to identify whether the overexpression of endogenous TBX2 was associated with cisplatin resistance in TBX2-driven cancers, TBX2 was silenced in a cisplatin-resistant breast cancer cell line (57). This demonstrated that TBX2 knockdown sensitized the cells to cisplatin by disrupting the ATM-CHK2-p53 signaling pathway (Fig. 1). Cell cycle analyses demonstrated that TBX2 knockdown led to a decrease in S-phase arrest, but robust G2/M arrest, which correlated with a reduction in phosphorylated CHK2 and p53 protein levels. This knockdown prevented DNA repair, and resulted in TBX2-deficient cells entering mitosis with damaged DNA and consequently undergoing mitotic catastrophe (57). These results suggest that targeting TBX2 in combination with typical chemotherapeutic drugs, including cisplatin, may improve the efficacy of current anticancer treatments.

Wnt/ $\beta$-catenin signaling pathway. The Wnt signaling pathway, a critical regulator of stem cell function, is involved in numerous aspects of embryonic development and controls homeostatic self-renewal in a number of adult tissues (58). Germline mutations in the Wnt signaling pathway cause several hereditary diseases, and somatic mutations in this signaling pathway are associated with cancer of the intestine, liver and a variety of other tissues (59). Therefore, the tightly regulated self-renewal mediated by the Wnt signaling pathway in stem and progenitor cells may be disrupted in cancer cells, allowing for malignant proliferation.

Using ChIP and reporter assays, a previous study identified TBX3 as a direct downstream regulatory target gene of the Wnt/ $\beta$-catenin signaling pathway that has been implicated in hepatocarcinogenesis (60). Furthermore, TBX3 transcription was activated by ectopic expression of $\beta$-catenin in mouse and human tumor cell lines (60) (Fig. 1). In addition, it has been shown that inhibition of TBX3 by small interfering RNAs (siRNAs) blocks $\beta$-catenin-mediated cell survival and renders cells sensitive to doxorubicin-induced apoptosis (60). Conversely, ectopic expression of TBX3 has been identified to inhibit apoptosis induced by $\beta$-catenin depletion (60). The results of this study reveal a role for TBX3 as a mediator of $\beta$-catenin-induced cell proliferation and regulator of hepatocarcinogenesis. Fong et al (61) demonstrated that TBX2 functions within the context of the Wnt signaling pathway to mediate cell migration in early embryonic development, indicating that TBX2 may be a target of the Wnt signaling pathway. However, whether TBX2 is a direct target of the Wnt signaling pathway in cancer cells remains unclear.

Akt serine/threonine kinase 3 (AKT)/TBX3/E-cadherin axis. The AKT family includes AKT1, AKT2 and AKT3, among which AKT3 is the predominant isoform $(62,63)$. There is growing evidence that AKT3 serves a key role in the proliferation, invasion and migration of melanomas (64). A previous study demonstrated that there is a synergistic co-operation between B-RAF-V600E and AKT3 in promoting melanoma development (65). In addition, TBX3 may be regulated by AKT3 (65). Notably, Peres et al (66) tested three TBX3 overexpressing melanoma cell lines, MM200, ME1402 and 501-mel, and observed a direct correlation between TBX3 mRNA and protein levels, suggesting that TBX3 may be upregulated at the transcriptional and post-transcriptional level in cancer. In addition, a direct positive correlation between phosphorylated AKT levels and TBX3 expression was identified through western blotting (66). Following AKT3 silencing by siRNA in ME1402 and MM200 melanoma cells in vertical growth phase (VGP), TBX3 protein levels were reduced (66). Subsequently, site-directed mutagenesis and in vitro kinase assays demonstrated that TBX3 was phosphorylated at Ser-720 by AKT3 in vitro (66). Furthermore, it was observed that the phosphorylation of S720 enhanced the ability of TBX3 to repress its target gene, E-cadherin (23). TBX3 and E-cadherin protein levels were found to be inversely correlated in ME1402 VGP melanoma cells (66), indicating that the AKT/TBX3/E-cadherin axis is involved in melanoma invasion and migration, and that TBX3 may function downstream of the AKT3 pathway (Fig. 1). Therefore, TBX3 may become a target gene for the treatment of advanced melanomas.

\section{TBX genes as targets for cancer therapy}

There is a lack of effective long-term cancer treatments. Therefore, there is a need for novel therapeutic targets for the treatment of cancer. The studies examined in the present review indicate that further studies on TBX genes and their protein products are important, and that any effective anticancer treatments must inhibit these genes. No other proteins have small repression domains (RD), so it may be possible to design a drug targeting the RD, and thus TBX genes, with a high specificity (67). This may block the function of TBX proteins in cancer cells. Furthermore, highly specific treatments have the advantage of possessing low toxicity and a minimal side-effect profile (68-70). Through increased understanding of the interaction between TBX proteins and their signaling partners in various types of cancer, it may be possible to develop successful TBX RD-specific inhibitors in the future.

\section{Conclusion}

In conclusion, the present review provides an overview of the roles of TBX genes in the development of various types of cancer, including tumor cell invasion and migration, anti-senescence and proliferation, tumorigenesis, EMT and tumorigenic signaling. In addition, the current review illustrates the potential to use TBX genes as targets for cancer therapy, which is currently being examined $(1,71)$. The theoretical rationale behind this is based on the fact that TBX genes are overexpressed in various types of cancer and function as potential oncogenes. An increased understanding of TBX genes and their protein products will accelerate the development of new cancer therapies targeting these genes.

\section{Acknowledgements}

The present review was supported by the National Nature Science Foundation of China (grant nos. 81372872, 81402215 and 
81320108022), the Nature Science Foundation of Tianjin (grant no. 16JCYBJC24100) and the program for Innovative University Research Teams in China (grant no. IRT14R40).

\section{References}

1. Lu J, Li XP, Dong Q, Kung HF and He ML: TBX2 and TBX3: The special value for anticancer drug targets. Biochim Biophys Acta 1806: 268-274, 2010

2. Abrahams A, Parker MI and Prince S: The T-box transcription factor Tbx2: Its role in development and possible implication in cancer. IUBMB Life 62: 92-102, 2010.

3. Takeuchi JK, Koshiba-Takeuchi K, Suzuki T, Kamimura M, Ogura K and Ogura T: Tbx5 and Tbx4 trigger limb initiation through activation of the Wnt/Fgf signaling cascade. Development 130 2729-2739, 2003

4. Kispert A and Hermann BG: The Brachyury gene encodes a novel DNA binding protein. EMBO J 12: 4898-4899, 1993.

5. MÜller CW and Herrmann BG: Crystallographic structure of the T domain-DNA complex of the Brachyury transcription factor. Nature 389: 884-888, 1997.

6. Ruvinsky I and Gibson-Brown JJ: Genetic and developmental bases of serial homology in vertebrate limb evolution. Development 127 $5233-5244,2000$

7. Tada $\mathrm{M}$ and Smith JC: T-targets: Clues to understanding the functions of T-box proteins. Dev Growth Differ 43: 1-11, 2001.

8. Papaioannou VE: The T-box gene family: Emerging roles in development, stem cells and cancer. Development 141: 3819-3833, 2014.

9. Stein RA: A new TBX gene linked to human disease. Clin Genet 76 23-24, 2009.

10. Naiche LA, Harrelson Z, Kelly RG and Papaioannou VE: T-box genes in vertebrate development. Annu Rev Genet 39: 219-239, 2005.

11. Morley RH, Lachani K, Keefe D, Gilchrist MJ, Flicek P, Smith JC and Wardle FC: A gene regulatory network directed by zebrafish No tail accounts for its roles in mesoderm formation. Proc Natl Acad Sci USA 106: 3829-3834, 2009.

12. Gentsch GE, Owens ND, Martin SR, Piccinelli P, Faial T, Trotter MW, Gilchrist MJ and Smith JC: In vivo T-box transcription factor profiling reveals joint regulation of embryonic neuromesodermal bipotency. Cell Rep 4: 1185-1196, 2013.

13. Lolas M, Valenzuela PD, Tjian R and Liu Z: Charting Brachyury-mediated developmental pathways during early mouse embryogenesis. Proc Natl Acad Sci USA 111: 4478-4483, 2014.

14. Papaioannou VE and Silver LM: The T-box gene family. Bioessays 20: 9-19, 1998.

15. Mowla S, Pinnock R, Leaner VD, Goding CR and Prince S: PMA-induced up-regulation of TBX3 is mediated by AP-1 and contributes to breast cancer cell migration. Biochem J 433: 145-153, 2011.

16. Lingbeek ME, Jacobs JJ and van Lohuizen M: The T-box repressors TBX 2 and TBX3 specifically regulate the tumor suppressor gene p14ARF via a variant T-site in the initiator. J Biol Chem 277: 26120-26127, 2002

17. Davenport TG, Jerome-Majewska LA and Papaioannou VE: Mammary gland, limb and yolk sac defects in mice lacking Tbx3, the gene mutated in human ulnar mammary syndrome. Development 130: 2263-2273, 2003.

18. Rowley M, Grothey E and Couch FJ: The role of Tbx 2 and Tbx 3 in mammary development and tumorigenesis. J Mammary Gland Bio Neoplasia 9: 109-118, 2004.

19. Fan W, Huang X, Chen C, Gray J and Huang T: TBX3 and its isoform $\mathrm{TBX} 3+2 \mathrm{a}$ are functionally distinctive in inhibition of senescence and are overexpressed in a subset of breast cancer cell lines. Cancer Res 64: 5132-5139, 2004

20. Li J, Weinberg MS, Zerbini L and Prince S: The oncogenic TBX3 is a downstream target and mediator of the TGF- $\beta 1$ signaling pathway. Mol Biol Cell 24: 3569-3576, 2013.

21. Peres J, Davis E, Mowla S, Bennett DC, Li JA, Wansleben S and Prince S: The highly homologous T-Box transcription factors, TBX2 and TBX3, have distinct roles in the oncogenic process. Genes Cancer 1: 272-282, 2010

22. Boyd SC, Mijatov B, Pupo GM, Tran SL, Gowrishankar K, Shaw HM, Goding CR, Scolyer RA, Mann GJ, Kefford RF, et al: Oncogenic B-RAF(V600E) signaling induces the T-Box3 transcriptional repressor to repress E-cadherin and enhance melanoma cell invasion. J Invest Dermatol 133: 1269-1277, 2013.
23. Rodriguez M, Aladowicz E, Lanfrancone L and Goding CR: Tbx3 represses E-cadherin expression and enhances melanoma invasiveness. Cancer Res 68: 7872-7881, 2008.

24. Mowla S, Pinnock R, Leaner VD, Goding CR and Prince S: PMA-induced up-regulation of TBX3 is mediated by AP-1 and contributes to breast cancer cell migration. Biochem $\mathrm{J} 433$ : 145-153, 2011.

25. Jackson D, Zheng Y, Lyo D, Shen Y, Nakayama K, Nakayama KI, Humphries MJ, Reyland ME and Foster DA: Suppression of cell migration by protein kinase Cdelta. Oncogene 24: 3067-3072, 2005.

26. Wang ZG, Delva L, Gaboli M,RiviR, Giorgio M,Cordon-Cardo C, Grosveld F and Pandolfi PP: Role of PML in cell growth and the retinoic acid pathway. Science 279: 1547-1551, 1998.

27. Gurrieri C, Capodieci P, Bernardi R, Scaglioni PP, Nafa K, Rush LJ, Verbel DA, Cordon-Cardo C and Pandolfi PP: Loss of the tumor suppressor PML in human cancers of multiple histologic origins. J Natl Cancer Inst 96: 269-279, 2004.

28. Martin N, Benhamed M, Nacerddine K, Demarque MD, van Lohuizen M, Dejean A and Bischof O: Physical and functional interaction between PML and TBX2 in the establishment of cellular senescence. EMBO J 31: 95-109, 2012.

29. Pomerantz J, Schreiber-Agus N, Liégeois NJ, Silverman A, Alland L, Chin L, Potes J, Chen K, Orlow I, Lee HW, et al: The Ink4a tumor suppressor gene product, p19Arf, interacts with MDM2 and neutralizes MDM2's inhibition of p53. Cell 92: 713-723, 1998.

30. Stott FJ, Bates S, James MC, McConnell BB, Starborg M, Brookes S, Palmero I, Ryan K, Hara E, Vousden KH and Peters G: The alternative product from the human CDKN2A locus, p14(ARF), participates in a regulatory feedback loop with p53 and MDM2. EMBO J 17: 5001-5014, 1998.

31. Prince S, Carreira S, Vance KW, Abrahams A and Goding CR: Tbx2 directly represses the expression of the p21(WAF1) cyclin-dependent kinase inhibitor. Cancer Res 64: 1669-1674, 2004.

32. Gitenay D and Baron VT: Is EGR1 a potential target for prostate cancer therapy? Future Oncol 5: 993-1003, 2009

33. Krones-Herzig A, Adamson E and Mercola D: Early growth response 1 protein, an upstream gatekeeper of the p53 tumor suppressor, controls replicative senescence. Proc Natl Acad Sci USA 100: 3233-3238, 2003

34. Redmond KL Crawford NT, Farmer H, D'Costa ZC, O'Brien GJ, Buckley NE, Kennedy RD, Johnston PG, Harkin DP and Mullan PB: T-box 2 represses NDRG1 through an EGR1-dependent mechanism to drive the proliferation of breast cancer cells. Oncogene 29: 3252-3262, 2010.

35. Zhu B, Zhang M, Byrum SD, Tackett AJ and Davie JK: TBX2 blocks myogenesis and promotes proliferation in rhabdomyosarcoma cells. Int J Cancer 135: 785-797, 2014

36. Ruas $M$ and Peters G: The p16INK4a/CDKN2A tumor suppressor and its relatives. Biochim Biophys Acta 1378: F115-F177, 1998.

37. Vance KW, Carreira S, Brosch G and Goding CR: Tbx2 is overexpressed and plays an important role in maintaining proliferation and suppression of senescence in melanomas. Cancer Res 65: 2260-2268, 2005.

38. Basson CT, Bachinsky DR, Lin RC, Levi T, Elkins JA, Soults J, Grayzel D, Kroumpouzou E, Traill TA, Leblanc-Straceski J, et al: Mutations in human TBX5 [corrected] cause limb and cardiac malformation in Holt-Oram syndrome. Nat Genet 15: 30-35, 1997.

39. He ML, Chen Y, Peng Y, Jin D, Du D, Wu J, Lu P, Lin MC and Kung HF: Induction of apoptosis and inhibition of cell growth by developmental regulator hTBX5. Biochem Biophys Res Commun 297: 185-192, 2002

40. Liu WK, Jiang XY and Zhang ZX: Expression of PSCA, PIWIL1, and TBX2 in endometrial adenocarcinoma. Onkologie 33: 241-245, 2010

41. Glozak MA, Sengupta N, Zhang X and Seto E: Acetylation and deacetylation of non-histone proteins. Gene 363: 15-23, 2005

42. Dokmanovic M and Marks PA: Prospects: Histone deacetylase inhibitors. J Cell Biochem 96: 293-304, 2005

43. Puri PL, Iezzi S, Stiegler P, Chen TT, Schiltz RL, Muscat GE, Giordano A, Kedes L, Wang JY and Sartorelli V: Class I histone deacetylases sequentially interact with $\mathrm{MyoD}$ and $\mathrm{pRb}$ during skeletal myogenesis. Mol Cell 8: 885-897, 2001.

44. Zhang X, Ozawa Y, Lee H, Wen YD, Tan TH, Wadzinski BE and Seto E: Histone deacetylase 3 (HDAC3) activity is regulated by interaction with protein serine/threonine phosphatase 4 . Genes Dev 19: 827-839, 2005. 
45. Pan LN, Lu J and Huang B: HDAC inhibitors: A potential new category of anti-tumor agents. Cell Mol Immunol 4: 337-343, 2007.

46. Hosford SR and Miller TW: Clinical potential of novel therapeutic targets in breast cancer: CDK4/6, Src, JAK/STAT, PARP, HDAC, and PI3K/AKT/mTOR pathways. Pharmgenomics Pers Med 7: 203-215, 2014

47. Yarosh W, Barrientos T, Esmailpour T, Lin L, Carpenter PM, Osann $\mathrm{K}$, Anton-Culver $\mathrm{H}$ and Huang T: TBX3 is overexpressed in breast cancer and represses p14 ARF by interacting with histone deacetylases. Cancer Res 68: 693-699, 2008.

48. Burgucu D, Guney K, Sahinturk D, Ozbudak IH, Ozel D, Ozbilim G and Yavuzer U: Tbx3 represses PTEN and is over-expressed in head and neck squamous cell carcinoma. BMC Cancer 12: 481, 2012.

49. Moral M and Paramio JM: Akt pathway as a target for therapeutic intervention in HNSCC. Histol Histopathol 23: 1269-1278, 2008.

50. Palena C, Polev DE, Tsang KY, Fernando RI, Litzinger M, Krukovskaya LL, Baranova AV, Kozlov AP and Schlom J: The human T-box mesodermal transcription factor Brachyury is a candidate target for T-cell-mediated cancer immunotherapy. Clin Cancer Res 13: 2471-2478, 2007.

51. Shimoda M, Sugiura T, Imajyo I, Ishii K, Chigita S, Seki K, Kobayashi Y and Shirasuna K: The T-box transcription factor Brachyury regulates epithelial-mesenchymal transition in association with cancer stem-like cells in adenoid cystic carcinoma cells. BMC Cancer 12: 377, 2012.

52. Grünert $S$, Jechlinger $M$ and Beug $H$ : Diverse cellular and molecular mechanisms contribute to epithelial plasticity and metastasis. Nat Rev Mol Cell Biol 4: 657-665, 2003.

53. Nieto MA: The snail superfamily of zinc-finger transcription factors. Nat Rev Mol Cell Biol 3: 155-166, 2002.

54. Bolós V, Peinado H, Pérez-Moreno MA, Fraga MF, Esteller M and Cano A: The transcription factor Slug represses E-cadherin expression and induces epithelial to mesenchymal transitions: A comparison with Snail and E47 repressors. J Cell Sci 116: 499-511, 2003

55. Yang J, Mani SA, Donaher JL, Ramaswamy S, Itzykson RA, Come C, Savagner P, Gitelman I, Richardson A and Weinberg RA Twist, a master regulator of morphogenesis, plays an essential role in tumor metastasis. Cell 117: 927-939, 2004

56. Wang B, Lindley LE, Fernandez-Vega V, Rieger ME, Sims AH and Briegel KJ: The T box transcription factor TBX2 promotes epithelial-mesenchymal transition and invasion of normal and malignant breast epithelial cells. PLoS One 7: e41355, 2012.

57. Wansleben S, Davis E, Peres J and Prince S: A novel role for the anti-senescence factor TBX2 in DNA repair and cisplatin resistance. Cell Death Dis 4: e846, 2013.
58. Reya $\mathrm{T}$ and Clevers $\mathrm{H}$ : Wnt signalling in stem cells and cancer. Nature 434: 843-850, 2005

59. Clevers H: Wnt/beta-catenin signaling in development and disease. Cell 127: 469-480, 2006.

60. Renard CA, Labalette C, Armengol C, Cougot D, Wei Y, Cairo S, Pineau $\mathrm{P}$, Neuveut $\mathrm{C}$, de Reyniès $\mathrm{A}$, Dejean $\mathrm{A}$, et al: $\mathrm{Tbx} 3$ is a downstream target of the Wnt/beta-catenin pathway and a critical mediator of beta-catenin survival functions in liver cancer. Cancer Res 67: 901-910, 2007.

61. Fong SH, Emelyanov A, Teh C and Korzh V: Wnt signalling mediated by $\mathrm{Tbx} 2 \mathrm{~b}$ regulates cell migration during formation of the neural plate. Development 132: 3587-3596, 2005.

62. Manning BD and Cantley LC: AKT/PKB signaling: Navigating downstream. Cell 129: 1261-1274, 2007.

63. Brazil DP, Park J and Hemmings BA: PKB binding proteins. Getting in on the Akt. Cell 111: 293-303, 2002.

64. Stahl JM, Sharma A, Cheung M, Zimmerman M, Cheng JQ, Bosenberg MW, Kester M, Sandirasegarane L and Robertson GP: Deregulated Akt3 activity promotes development of malignant melanoma. Cancer Res 64: 7002-7010, 2004.

65. Cheung M, Sharma A, Madhunapantula SV and Robertson GP: Akt3 and mutant V600E B-Raf cooperate to promote early melanoma development. Cancer Res 68: 3429-3439, 2008.

66. Peres J, Mowla S and Prince S: The T-box transcription factor, $\mathrm{TBX} 3$, is a key substrate of AKT3 in melanomagenesis. Oncotarget 6: 1821-1833, 2015.

67. Paxton C, Zhao H, Chin Y, Langner K and Reecy J: Murine Tbx2 contains domains that activate and repress gene transcription. Gene 283: 117-24, 2002.

68. Zhou Y, Du W, Koretsky T, Bagby GC and Pang Q: TAT-mediated intracellular delivery of NPM-derived peptide induces apoptosis in leukemic cells and suppresses leukemogenesis in mice. Blood 112: 2474-2483, 2008

69. Fåhraeus R, Laín S, Ball KL and Lane DP: Characterization of the cyclin-dependent kinase inhibitory domain of the INK4 family as a model for a synthetic tumour suppressor molecule. Oncogene 16: 587-596, 1998.

70. Bonfanti M, Taverna S, Salmona M, D'Incalci $M$ and Broggini M: p21WAF1-derived peptides linked to an internalization peptide inhibit human cancer cell growth. Cancer Res 57: 1442-1446, 1997.

71. Douglas NC and Papaioannou VE: The T-box transcription factors TBX2 and TBX3 in mammary gland development and breast cancer. J Mammary Gland Biol Neoplasia 18: 143-147, 2013. 